\title{
PREDICTION OF THERMALEFFECTS OF MAGNITUDE FOR HRAM EVENT IN FUEL-FILLED TANK USING THE RAYLEIGH-PLESSET EQUATION
}

\author{
T. FOUREST ${ }^{1}$, M. ARRIGONI ${ }^{2}$, E. DELETOMBE ${ }^{1}$, J. DUPAS ${ }^{1} \&$ J-M. LAURENS ${ }^{2}$ \\ ${ }^{1}$ ONERA - The French Aerospace Lab, F-59045, Lille, France \\ ${ }^{2}$ ENSTA-Bretagne, FRE CNRS 3744 - IRDL, F-29806, Brest, France.
}

\begin{abstract}
To reduce the vulnerability of both civilian and military aircraft, it is important to take the hydrodynamic ram (HRAM) effect into account when designing their fuel tanks. HRAM is especially dangerous for liquid- filled thin walled lightweight structures that cannot be armoured due to weight penalty reasons. However, the response of the tank structure during HRAM events depends on a coupling model between fluid and structure. Water is generally used as a liquid candidate for experimental observations of HRAM, since it is a safe and affordable solution. However, its characteristics in thermal transfers are far different from the ones of hydrocarbons, and it may influence the bubble behaviour and thus its resulting loading on the tank walls. A good understanding of all these aspects is still needed to enhance the tank designs. Similarities in bubble behaviour between HRAM and underwater explosion situations were observed in recent high-speed tank penetration/water entry experiments. A confined version of the Rayleigh-Plesset equation - which is classically used for bubble dynamics analysis (including underwater explosion) - has been previously proposed to simulate a bubble created by an HRAM event. The work the presented work is a first attempt to the estimation of the influence of thermal effects in HRAM processes, by using the Rayleigh-Plesset equation in confined regime.

Keywords: ballistic impact, cavitation, fuel-filled tank, hydrodynamic ram, Rayleight-Plesset equation, thermal effects.
\end{abstract}

\section{INTRODUCTION}

In the event of impact of high-speed/high-energy projectiles on liquid filled tanks, the container may suffer large hydrodynamic loads that could possibly rupture the entire structure. This impact scenario is referred as hydrodynamic ram (HRAM). There is an increasing need of tools for physical understanding of the effects which occur during a HRAM event as well in the civil domain as for the military aircraft design (vulnerability requirement). Indeed physical understanding of HRAM dynamics would permit to implement its description in predictive models that will make the structural design of tanks more reliable with respect to this specific threat.

Ball described the HRAM event that is generally characterized by four stages - the shock stage, the drag stage, the cavity growth and collapse stages [1]. These stages and the associated loads are illustrated in Fig. 1.

The first stage is the impact of the projectile on the container wall and the penetration in the liquid. Consequent to this impact, a quasi hemispherical shock wave is emitted in the liquid. Then, there is a stage of drag of the projectile in the liquid, during which the projectile transmits most of its kinetic energy to the surrounding liquid, set it in motion, and generates a 


\begin{tabular}{cccc}
\hline $\begin{array}{c}\text { Entry wall } \\
\text { penetration }\end{array}$ & $\begin{array}{c}\text { Drag of } \\
\text { projectile }\end{array}$ & Cavity growth & $\begin{array}{c}\text { Cavity } \\
\text { collapse }\end{array}$ \\
\hline Fluid shock & Drag pressure & $\begin{array}{c}\text { Cavity growth } \\
\text { pressure }\end{array}$ & $\begin{array}{c}\text { Collapse fluid } \\
\text { shock }\end{array}$ \\
\hline & & &
\end{tabular}

Figure 1: General scenario of hydrodynamic ram event in liquid-filled tank.

cavity in its wake. Due to the high velocity of the impact, the cavity is not only filled with air but also with liquid vapour, especially when the wake of the projectile undergoes a sealing as in Deletombe et al. [2]. This fluid movement and the resulting pressure might continue for a long time after the exit of the projectile. During the implosion of the cavity, a secondary shock wave is emitted that could also contribute to the damage of the container. Numerical simulations with adhoc models allow observing and understanding the event of HRAM. Some attempts of numerical simulations can be found in the open literature Varas et al. [3], relying on Euler Lagrange or particle type methods. However, the whole sequence of events up to the collapse (that might take up to $30 \mathrm{~ms}$ ) is not entirely simulated. Plus these simulations do not deal with the phenomenon of tumbling that occurs for many cases of impacts of real projectiles. This phenomenon which is illustrated in Fig. 1, influences strongly the shape of the cavity (more spherical for tumbling cases) and the pressure applied onto the container (possibly five times superior according to Bless [4]).

An alternative approach to the use of numerical simulation is to study this complex phenomenon with analytical tools. Pioneer studies on the subject were carried out by Stepka and Morse [5]. They performed numerous experimental measurements during high-speed projectile impacts on liquid-filled tank. They identified the factors that affect the loading during a hydrodynamic ram event. These factors can be organised according to the three media: the projectile (shape, size and material), the tank wall (the thickness, material, pre-stress and protective structure) and the liquid properties. They tried to determine the influences of the different parameters on the survivability of tanks. However, they could not clearly establish a correlation that would embrace the effects of all parameters.

Recent experimental results, in particular those presented in Deletombe et al. [2], permitted to observe the evolution of the bubble created in the wake of a tumbling projectile. Since the bubble dynamics is due to a prompt release of a high energy, like during underwater explosions Cole [6], a RayleighPlesset like model is chosen to describe this phenomenon Fourest et al. [7].

In all the experimental tests presented in the literature, impacts have been done on waterfilled tank since water is a 'safe' and affordable liquid candidate. It is generally assumed that studying HRAM in a water-filled tank gives insight on this phenomenon in fuel-filled tanks. However, water is not a representative liquid in terms of heat transfers with respect to hydrocarbons. It is crucial to clarify the influence of the heat transfer on the bubble dynamics, and to take it into account in available models if necessary.

First, the confined Rayleigh-Plesset equation will be presented and applied to simulate two ballistic impact tests in water-filled tanks. Then, once it has been shown that the dynamics of the gas bubbles generated during ballistic impacts in liquid can be described using Rayleigh-Plesset type equation, two classic thermal effects indicators related to the 
Rayleigh-Plesset equation will be calculated on several liquids. In this way, it is possible to estimate the amount of thermal effects that can be expected to participate at the bubble dynamics consecutively to impacts in fuel tanks. To the knowledge of authors, this approach has not been applied to aeronautic fuel materials previously.

\section{STUDIED CASES}

The Rayleigh-Plesset approach has been used for two cases: the first one is a $7.62 \mathrm{~mm}$ NATO ballistic impact in a generic Airbus-Group Innovation closed water-filled tank. The second case is a ballistic shot in a large ONERA/DAAP pool. In both cases, the projectile trajectory is normal to the entry wall of the tank or free surface of liquid. These experiments are reported in Deletombe et al. [2]. These two tests correspond respectively to a confined tank and a larger domain. The pool dimensions are approximately $22 \times 1.5 \times 1.5 \mathrm{~m}^{3}$ and the tank dimensions are $0.3 \times 0.54 \times 0.66 \mathrm{~m}^{3}$.

\section{CONFINED RAYLEIGH-PLESSET EQUATION}

Early studies on bubble dynamics began with Lord Rayleigh [8] who examined the pressure prediction during the collapse of a spherical bubble, assuming that the surrounding liquid is incompressible and inviscid, and that surface tension forces are negligible. His work was extended by Plesset [9], who derived the second-order non-linear ordinary differential equation for the time-dependent bubble radius evolution, which became the well-known Rayleigh-Plesset equation for bubble dynamics. Improvements of this equation have been proposed by numerous authors, an example is the work of Fujikawa and Akamatsu [10]. These improvements mainly concern effects that are important at the end of the collapse stage for water cavitation: liquid compressibility, thermal effects and the effect of the non-equilibrium of liquid-vapour transformation.

\subsection{Presentation of the confined Rayleigh-Plesset equation}

A modification of the classic Rayleigh-Plesset equation has been proposed by the authors in Fourest et al. [7] to account for confinement effects without changing the method of resolution. To obtain this equation and use it to predict HRAM bubble dynamics, a spherical gas bubble in a spherical finite domain of liquid is first considered, and the following assumptions are made:

- Spherical deformation of the bubble interface;

- Instantaneous energy transfer from kinetic energy of the bullet to the liquid;

- Gravity effects are negligible;

- Idealised case of zero mass transport across the bubble interface is considered,

- Dynamic viscosity and surface tension effects are negligible due to the large dimensions of the bubbles,

- An initial amount of non-condensable gas (here air) is considered, in first approach, its behaviour is assumed to be adiabatic and behaves as an ideal gas.

- The liquid domain is considered to be of finite dimensions;

- An elastic structural confinement is added by means of a spherical shell.

The equation of mass conservation for a radial movement is expressed in spherical coordinates. It reduces due to the assumptions to (1). 


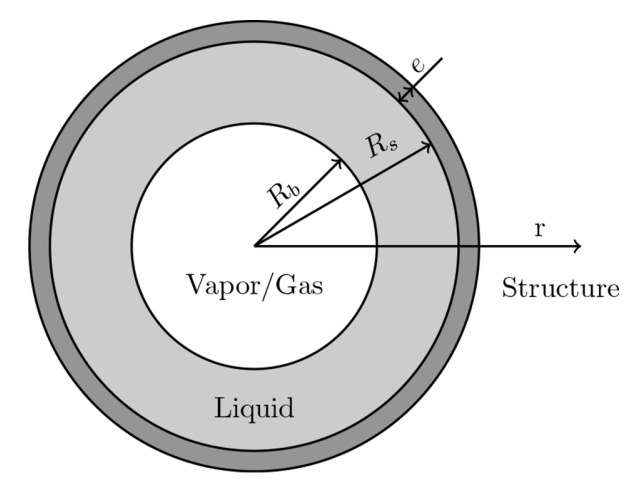

Figure 2: System considered in the proposed confined Rayleigh-Plesset equation.

$$
r^{2} \dot{r}=R_{b}^{2} \dot{R}_{b}
$$

with $R b$ the radius of the bubble and $\dot{r}$ the radial speed of the liquid at radius $r$. Using the previous assumptions, the equation of conservation of momentum in the radial direction is reduced to (2).

$$
\ddot{r}+\dot{r} \frac{\partial \dot{r}}{\partial r}+\frac{1}{\rho} \frac{\partial P}{\partial r}=0
$$

It is considered that the centre of the bubble corresponds to the centre of the container. The initial external radii $R_{s 0}$ of the liquid domains were defined as the radii that yield the same volume as the tested containers (respectively $50 \mathrm{~m}^{3}$ for the pool and $7.7 .10^{-2} \mathrm{~m}^{3}$ for the tank). The behaviour of the liquid is assumed to be incompressible, hence the current radius of the elastic spherical container $R_{s}$ is related to the radius of the bubble (3):

$$
\left.R s=R_{s 0}^{3}+R_{b}^{3}-R_{b 0}^{3}\right)^{1 / 3}
$$

Integrating (2) between $R_{s}$ and $R_{b}$, using (1) and defining $\Lambda=R_{b} / R_{s}$, the same equation as in Obreschkow et al. [11] is found (4):

$$
R_{b} \ddot{R}_{b}+\frac{3}{2} \dot{R}_{b}^{2}+\frac{P_{s}(t)-P_{b}(t)}{\rho}-2 \dot{R}_{b}^{2} \Lambda-R_{b} \ddot{R}_{b} \Lambda+\frac{1}{2} \dot{R}_{b}^{2} \Lambda^{4}=0
$$

with $P_{s}$ the pressure at the interface between the liquid and the structural spherical container. $\Lambda$ is then defining a geometrical parameter with respect to the finite size of the considered fluid domains.

A similar equation to the Rayleigh-Plesset equation is obtained with the addition of three terms that vanish when $R_{s}=\infty$ (infinite medium).

Another relation between the pressure applied on the wall and the structural sphere response is needed. If the behaviour of the containers is assumed elastic and linear, a good approximation of the relationship is obtained by assuming proportionality between the variation of pressure on the sphere wall $P_{s}-P_{s 0}$ and the variation of the internal volume of the sphere $V-V_{0}$. This coefficient of proportionality will be hereafter called confinement parameter, and denoted $\alpha$ in (5). 


$$
P_{s}-P_{s 0}=a \cdot\left(V-V_{0}\right)
$$

\subsection{Application of the confined Rayleigh-Plesset equation to HRAM in water-filled tank}

The initial conditions of the Rayleigh-Plesset equation determined from the ONERA experiments are linked to the initial time chosen for the analysis (when a bubble cavity reasonably appears). To choose the starting times for Rayleigh-Plesset simulations, energetic considerations are used, for example, when the liquid initial kinetic energy in Rayleigh-Plesset equation is equal to the theoretical initial kinetic energy of the projectile that created the bubble (approximately $3.5 \mathrm{~kJ}$ in the pool and $2.9 \mathrm{~kJ}$ in the tank). It has been observed that it corresponds approximately to the beginning of the growth stage of the bubble cavity in the tests. As the energetic partition between the kinetic energy transferred to the liquid and the energy dissipated by the deformation of the projectile is not known, no dissipative phenomena are considered here: the whole projectile kinetic energy is assumed to be transferred to the liquid. The amount of kinetic energy of the liquid is calculated using the assumption of incompressibility of the liquid (6) is:

$$
E_{k}=2 \pi \rho R_{b}^{4} \dot{R}_{b}^{2}\left(\frac{1}{R_{b}}-\frac{1}{R_{s}}\right)
$$

In this work, the values of the confinement parameters are obtained by calibration. These values are denoted $\alpha_{\text {calib }}$.

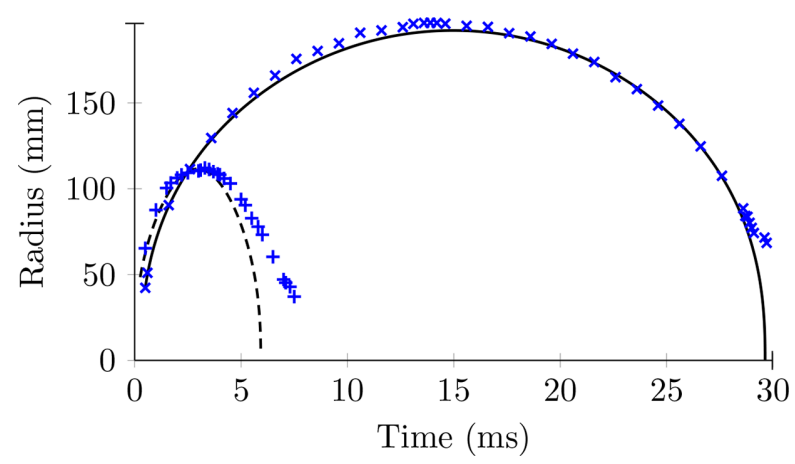

Figure 3: Radius evolution in tank test $(+)$, pool test $(\times)$ and predicted with confined RP in tank (---) and pool (-) with $\alpha_{\text {calib }}$.

Table 1: Numerical values of the initial conditions used for each case in confined RayleighPlesset simulations.

\begin{tabular}{lcccccc}
\hline Case & $\begin{array}{c}P_{\mathrm{bo}} \\
(\mathrm{MPa})\end{array}$ & $\begin{array}{c}t_{0} \\
(\mathrm{~ms})\end{array}$ & $\begin{array}{c}R_{\mathrm{b} 0} \\
(\mathrm{~mm})\end{array}$ & $\begin{array}{c}\dot{R}_{\mathrm{b} 0} \\
(\mathrm{~mm} \cdot \mathrm{ms}-1)\end{array}$ & $\begin{array}{c}R_{\mathrm{s} 0} \\
(\mathrm{~mm})\end{array}$ & $\begin{array}{c}\alpha_{\text {calip }} \\
\left(\mathrm{MPaa} . \mathrm{mm}^{-3}\right)\end{array}$ \\
\hline Pool & $8.1 .10^{-3}$ & 0.5 & 42.3 & 86.44 & 2,200 & 1.5 \\
Tank & $2.23 .10^{-3}$ & $0.26 \mathrm{~ms}$ & 48.5 & 70.6 & 264 & 150 \\
\hline
\end{tabular}


The modified Rayleigh-Plesset equation is solved with a Runge-Kutta forth order method. The coefficients $\alpha_{\text {calib }}$ have been chosen to obtain good agreement in amplitude between the experimental radii and those obtained numerically (Fig. 3). Table 1 summarises the initial conditions used for these simulations. $\alpha_{\text {calib }}=1.5 \mathrm{MPa} \mathrm{mm}^{-3}$ and $\alpha_{\text {calib }}=150 \mathrm{MPa} \cdot \mathrm{mm}-3$ for the pool and tank, respectively.

It can be seen from Fig. 3 that the experimental bubble radii can be reasonably well described using a Rayleigh-Plesset type equation.

The agreement is better for the pool test, in which the bubble created in the experiment is more spherical (Deletombe et al. [2]). The application of the confined Rayleigh-Plesset equation is here presented with a calibration of the structure response, but it has been shown in Fourest et al. [12] that the value of the confinement parameter can be reasonably well approximated using analytical models on the plates of the container.

\section{CALCULATION OF RAYLEIGH-PLESSET-BASED THERMAL INDICATOR FOR HRAM IN FUEL TANK}

According to classical thermodynamics, cavitation occurs at the edge of the saturation domain from liquid to gaseous state of water in its phase diagram. This happens when the pressure is lower than the vapour pressure, which is a function of the liquid temperature. In usual atmospheric conditions, only a small amount of heat is needed to generate a large volume of vapour, so the vaporisation is assumed isothermal (as in the previous study). However, in certain cases the heat transfer needed for this change of phase is large enough that a significant drop in the local temperature $T_{c}$ of the liquid is achieved with respect to the temperature of the liquid far from the cavity $T_{\infty}$. Then, this local drop in the liquid temperature results in a drop in the vaporisation pressure that facilitates the vaporisation process. The difference in temperature $T_{\infty}-T_{c}$ is called thermal delay in cavitation, because generally this phenomenon results in longer time for the oscillations of the bubble. It also results, according to Grazia et al. [13], in larger bubble radius. It is clear that if this phenomenon influences the bubble dynamics, it also influences the hydrodynamic loads applied on the structure during this phenomenon. So it is important to determine whether this phenomenon occurs for cavitation in aeronautic fuel materials, to take it into account if necessary.

\subsection{Characteristics of usual aeronautic fuel materials}

In this study, two fuel materials used in aeronautics are investigated: the Jet-A, which is one of the most common fuels used in civilian aeronautic and an aviation gasoline, which is more rarely used but has quite different characteristics. The behaviours of these liquids are compared with two references: water for which there is no thermal effect in cavitation at

Table 2: Numerical values of liquid thermal properties at the studied temperature (Part 1).

\begin{tabular}{lccccc}
\hline Liquid & $\begin{array}{c}\mathrm{T} \\
\left({ }^{\mathrm{O}} \mathrm{K}\right)\end{array}$ & $\begin{array}{c}\rho_{l} \\
\left(\mathrm{~kg} \cdot \mathrm{m}^{-3}\right)\end{array}$ & $\begin{array}{c}\rho_{v} \\
\left(\mathrm{~kg} \cdot \mathrm{m}^{-2}\right)\end{array}$ & $\begin{array}{c}v_{l} \\
\left(\mathrm{~mm} 2 \cdot \mathrm{s}^{-1}\right)\end{array}$ & $\begin{array}{c}S \\
\left(\mathrm{~N}^{-} \mathrm{m}^{-1}\right)\end{array}$ \\
\hline Water & 293 & 1,000 & $17.2 \cdot 10^{-2}$ & 1.007 & 0.0728 \\
Jet-A & 293 & 810 & $17.7 \cdot 10^{-2}$ & 1.75 & 0.023 \\
Av. Gas. & 293 & 700 & 0.81 & 0.65 & 0.020 \\
Hydrogen & 22 & 69 & 2.01 & 0.017 & $2 \cdot 10^{-3}$ \\
\hline
\end{tabular}


Table 3: Numerical values of liquid thermal properties at the studied temperature (Part 2).

\begin{tabular}{lcccc}
\hline & $\mathrm{T}$ & $\mathrm{L}_{e v}$ & $\mathrm{C}_{\mathrm{pl}}$ & $\mathrm{k}_{l}$ \\
Liquid & $\left({ }^{\mathrm{O}} \mathrm{K}\right)$ & $\left(\mathrm{kJ} \cdot \mathrm{kg}^{-1}\right)$ & $\left(\mathrm{kJ} \cdot \mathrm{kg}^{-1} \cdot \mathrm{K}^{-1}\right)$ & $\left(\mathrm{W} \cdot \mathrm{m}^{-1} \cdot \mathrm{K}^{-1}\right)$ \\
\hline Water & 293 & $2,454.3$ & 4.182 & 0.6 \\
Jet-A & 293 & 363 & 1.95 & 0.115 \\
Av. Gas. & 293 & 387 & 2.23 & 0.115 \\
Hydrogen & 22 & 447 & 11 & 0.12 \\
\hline
\end{tabular}

$T=273^{\circ} \mathrm{K}$ and hydrogen at $T=22^{\circ} \mathrm{K}$ for which there is large thermal effect Brennen [14]. A large number of numerical values of liquid thermal properties are summarized in Tables 2 and 3. The characteristics are extracted from the Handbook of Aviation Fuel Properties [15] and from other sources: Shepherd et al. [16, 17], Woodrow [18] for the fuel properties, from Franc et al. [19] for the water properties and from Jensen et al. [20] for the hydrogen properties.

\subsection{Indicator of thermal effects}

It has been shown previously that the dynamics of the cavity bubble created in the wake of a projectile can be modelled using a Rayleigh-Plesset type equation. The rigorous method to solve the bubble dynamics with thermal effects requires to solve simultaneously the Rayleigh-Plesset equation and the equation of heat diffusion. However, in first approach, it is common to rely on thermal effects indicators for bubble dynamics analysis that are related to the Rayleigh-Plesset equation as in Brennen [14] and Grazia et al. [13]. Two classic ones are used to estimate the importance of thermal effects on hydrodynamic ram bubble dynamics. The first one is $\Delta T$, which is the temperature drop required for the vaporisation of a volumetric unit of vapour, and $\Sigma$, that is defined by Brennen [14] in relation to a characteristic time $t_{c}$ at which the terms affected by thermal effects become as important as the other terms in the Rayleigh-Plesset equation, which means that the change of temperature during cavitation really influences the bubble dynamics.

The two indicators and the associated characteristic time are presented in (7), (8), and (9).

$$
\begin{gathered}
\Delta T=\frac{\rho_{v} L_{e v}}{\rho_{l} C_{p l}} \\
\Sigma=\frac{\rho_{v}^{2} L_{e v}^{2}}{\rho_{l}^{3 / 2} C_{p l}^{1 / 2} \cdot T_{\infty} \cdot k^{1 / 2}} \\
t_{c}=\frac{(P v-P \infty)}{\rho_{l}} \cdot \frac{1}{\sum^{2}}
\end{gathered}
$$

where $T_{\infty}$ is the temperature of the liquid far from the cavity, $\rho_{l}$ the liquid density, $\rho_{v}$ the vapour density, $v_{l}$ the kinematic viscosity, $S$ the surface tension, $L_{e v}$ the latent heat of vapori- 
Table 4: Numerical values of $\Delta T, \Sigma$ and $t c$ for the different liquids at the studied temperature.

\begin{tabular}{lcccc}
\hline Liquid & $\mathrm{T}\left({ }^{\circ} \mathrm{K}\right)$ & $\begin{array}{c}\Delta \mathrm{T} \\
\left({ }^{\circ} \mathrm{K}\right)\end{array}$ & $\begin{array}{c}\sum \\
\left(\mathrm{m} . \mathrm{s}^{3 / 2}\right)\end{array}$ & $\begin{array}{c}t_{c} \\
(\mathrm{~s})\end{array}$ \\
\hline Water & 293 & 0.01 & 3.84 & 10 \\
Jet-A & 293 & 0.004 & 0.41 & $10^{3}$ \\
Av. Gas. & 293 & 0.36 & 1131 & $10^{-5}$ \\
Hydrogen & 22 & 1.18 & $1.76 .10^{6}$ & $10^{-10}$ \\
\hline
\end{tabular}

sation, $C_{p l}$ the specific heat, and $k_{l}$ is defined as the ratio of heat capacities at constant pressure over constant volume.

The numerical values of these indicators and of the corresponding characteristic time of the calculated $\Sigma$ are presented in Table 4 for the liquids studied in the present work.

The numerical values of $\Delta T$ and $\Sigma$ for the Jet-A material are inferiors to the ones of the water, so it indicates that there is no thermal effect for cavitation in the Jet-A material, which is the most common fuel material for aeronautic civilian applications. So the use of impacts in waterfilled tanks as references to study the hydrodynamics ram phenomenon in fuel containers seems justified from this viewpoint. However, there may be some thermal effects for the cavitation in the aviation gasoline fuel for which the value is higher than for water but lower than for hydrogen. In addition, the value of $t_{c}$ is several order of magnitude inferior to the bubble period in this case (see Fig. 3). It also means that the thermal transfer has the time to influence the bubble dynamics. So, one should consider with care results obtained with a model that does not take the thermal effects into account to model a ballistic impact in this liquid.

\section{CONCLUSION}

In the present study, thermal effects that could occur during ballistic impacts on fuel tanks are investigated. Most of the work presented in the literature on ballistic impacts on liquid-filled tanks deal with impact on water-filled containers. However, when it comes to the modelling of this phenomenon, it is not clear if differences in the behaviour between water and fuel materials would modify the suitable models. In particular, thermal effects have been identified as a possible source of differences in the models needed to simulate impacts in water and fuel-filled tanks.

A more direct way to answer this question could be to compare isothermal numerical simulations (as done for impacts on water-filled containers) with experimental results for impacts on fuel-filled containers. However, such experimental reference is not available in the open literature and performing such tests is not straightforward due to additional security issues with respect to impact on water-filled tanks. In addition to this experimental difficulty, the most advanced numerical models available have not yet been fully physically justified, even if they seem to reproduce with a reasonable accuracy experimental results for impacts on water-filled tank (Varas et al. [3]). So if differences were obtained between the experiments and numerical simulations, it would be uncertain to attribute these differences to the sole influence of thermal effects.

To deal with all these problems, an alternative is proposed in the present work to calculate classical thermal effects indicators. These indicators are related to the use of the Rayleigh-Plesset equation for the analysis of cavitation bubbles. To justify the use of these indicators, first a confined version of the Rayleigh-Plesset equation has been applied to two 
cases of impact on water-filled tanks. The obtained results tend to show that a Rayleigh-Plesset analysis can be justified for ballistic impact in liquid-filled tanks. Then two classic thermal effects indicators have been calculated for four liquids. First for water at ambient temperature for which there are no thermal effect expected and for hydrogen at a low temperature at which there are large thermal effects that need to be modelled (Brennen [14]). Then, it has been applied to Jet-A which is one of the most common fuels used in civilian aeronautic and to an aviation gasoline, which is more rarely used but has quite different characteristics. The conclusion of this study is that no thermal effects are expected for cavitation in Jet-A, so the same isothermal numerical simulations performed on water might be used to simulate impacts on Jet-A filled tanks. However, some thermal effects are predicted for cavitation in the aviation gasoline. The modification that these thermal effects might have on the bubble dynamics and subsequent hydrodynamic loads has not been determined in this study. So a prolongation of this study would be to perform confined Rayleigh-Plesset simulation coupled with the resolution of the heat diffusion equation to estimate this modification.

\section{REFERENCES}

[1] Ball, R., The fundamental of aircraft combat survivability analysis and design. AIAA, 1985. http://dx.doi.org/10.2514/4.861239

[2] Deletombe, E., Fabis, J., Dupas, J. \& Mortier, J.M., Experimental analysis of $7.62 \mathrm{~mm}$ hydrodynamic ram in containers. Journal of Fluids and Structures, 37, pp. 1-21, 2013. http://dx.doi.org/10.1016/j.jfluidstructs.2012.11.003

[3] Varas, D., Zaera, R. \& L'opez-Puente, J., Numerical modelling of the hydrodynamic ram phenomenon. International Journal of Impact Engineering, 36, pp. 363-374, 2009. http://dx.doi.org/10.1016/j.ijimpeng.2008.07.020

[4] Bless, S., Fuel tank survivability for hydrodynamic ram induced by high-velocity fragments. Part I experimental result and design summary. Technical Report AFFDLTR-78-184, Part I, University of Dayton Research Institute, 1979.

[5] Stepka, F. \& Morse, C., Preliminary investigation of catastrophic fracture of liquidfilled tanks impacted by high velocity particles. Technical Report D-1537, NASA, Cleveland, Ohio, USA, 1963.

[6] Cole, R., Underwater Explosions, Princeton University Press: Princeton, pp. 7-13, 2845,102-109,114-126,425-426, 1945.

[7] Fourest, T., Laurens, J.M., Deletombe, E., Dupas, J. \& Arrigoni, M., Analysis of bubbles dynamics created by hydrodynamic ram in confined geometries using the rayleighplesset equation. International Journal of Impact Engineering, 73, pp. 66-74, 2014. http://dx.doi.org/10.1016/j.ijimpeng.2014.05.008

[8] Rayleigh, L., On the pressure developed in a liquid during the collapse of a spherical cavity. Philosophical Magazine Series 6, 34(200), pp. 94-98, 1917. http://dx.doi.org/10.1080/14786440808635681

[9] Plesset, M., The dynamics of cavitation bubbles. Journal of Applied Mechanics, 16, pp. 277-282, 1949.

[10] Fujikawa, S. \& Akamatsu, T., Effects of the non-equilibrium condensation of vapour on the pressure wave produced by the collapse of a bubble in liquid. Journal of Fluid Mechanics, 97(03), pp. 481-512, 1980.

http://dx.doi.org/10.1017/S0022112080002662 
[11] Obreschkow, D., Kobel, P., Dorsaz, N., de Bosset, A., Nicollier, C. \& Farhat, M., Cavitation bubble dynamics inside liquid drops in microgravity. Physical Review Letter, 97, 2006.

http://dx.doi.org/10.1103/physrevlett.97.094502

[12] Fourest, T., Laurens, J.M., Deletombe, E., Dupas, J. \& Arrigoni, M., Confined rayleighplesset equation for hydrodynamic ram analysis in thin-walled containers under ballistic impacts. Thin-Walled Structures, 86, pp. 67-72, 2015.

http://dx.doi.org/10.1016/j.tws.2014.10.003

[13] Grazia De Giorgi, M., Bello, D. \& Ficarella, A., Analysis of thermal effects in a cavitating orifice using rayleigh equation and experiments. Journal of Engineering for Gas Turbines and Power, 132(9), 2010. http://dx.doi.org/10.1115/1.4000367

[14] Brennen, C., Cavitation and Bubble Dynamics, Oxford University Press, 1995.

[15] Handbook of aviation fuel properties, Technical Report ADA132106, Coordinating Research Council, 1983.

[16] Shepherd, J., Krok, J. \& Lee, J.J., Jet a explosion experiements: Laboratory testing. Technical Report FM97-5, Graduate Aeronautical Laboratories, California Institute of Technology, 1997.

[17] Shepherd, J., Nuyt, C. \& Lee, J.J., Flash point and chemical composition of aviation kerosene (jet a). Technical Report FM99-4, Graduate Aeronautical Laboratories, California Institute of Technology, 2000.

[18] Woodrow, J. \& Seiber, J.N., The laboratory characterisation of fuel vapor under simulated flight conditions. Technical Report NTSB12-97-SP-0255, Center for Environmental Sciences and Engineering, 1997.

[19] Franc, J., Avellan, F., Belahadji, B., Billard, J., on Marjollet, L.B., Fr'echou, D., Fruman, D., Karimi, A., Kueny, J. \& Michel, J., La Cavitation, m'ecanisme, Presse Universitaire de Grenoble: Grenoble, France, pp. 63-95, 1995.

[20] Jensen, J., Tuttle, W., Stewart, R., Brechna, H. \& Prodell, A., Brookhaven national laboratory selected cryogenic data notebook, volume I, section I-IX. Technical Report BNL 10200-R, Vol. I, Brookhaven National Laboratory, 1980. 